\title{
Abuso de fármacos en medio sanitario: programas de tratamiento
}

\section{Substance-related abuse in health personnel: treatment programs}

\author{
Inma Caballero Aranda ${ }^{1,2,3}$, MP. Sevilla Lerena $a^{1,2,3}$ \\ 1. Instituto Navarro de Salud Laboral. Navarra. España. \\ 2. Unidad Docente de Medicina del Trabajo de Navarra. España. \\ 3. Unidad Docente de Medicina del Trabajo de la Comunidad de Madrid. España.
}

Recibido: 23-01-14

Aceptado: 24-02-14

\section{Correspondencia}

Inma Caballero Aranda

Instituto Navarro de Salud Laboral

Pol. de Landaben

Calle E, 31012 Pamplona. España.

Correo electrónico: icabara@yahoo.com

Resumen

Introducción: El colectivo sanitario tiene unas especiales características de carga de trabajo, estrés, turnicidad y jornada prolongada. Esto unido al fácil acceso a fármacos en su medio laboral, les hace susceptibles a posibles adicciones farmacológicas. Estas adicciones también suponen un riesgo para la salud de terceros. Existen diferentes programas de rehabilitación para conseguir la reincorporación de estos profesionales.

Objetivos. Conocer la situación actual de las adicciones a fármacos, los programas de rehabilitación y sus resultados en el personal sanitario.

Materiales y métodos: Se realizaron búsquedas bibliográficas en: MEDLINE (PUBMED), Scopus, Web of Knowledge (WOK), IBECS, LILACS, SCIELO y literatura gris.

Resultados: Se incluyeron un total de nueve artículos científicos de diversa procedencia. Los niveles de evidencia encontrados fueron bajos. Existen programas de rehabilitación y reinserción con resultados prometedores.

Med Segur Trab (Internet) 2014; 60 (235) 455-469

Palabras clave: Programas de tratamiento, abuso de sustancias, personal sanitario.

Abstract

Introduction: Health personnel have some unique characteristics related with workload, stress, work shifts and long work hours. All these factors, as well as the easy access to drugs in their job, make them candidates for possible substance-related abuse. These addictions are also a risk for the health of a third group: the patients. There are diverse treatment programs with a single objective: to obtain the reincorporation of these professionals.

Objectives: To know the current situation of substance related abuse, the treatment programs and their results with the health personnel. 
Methods: Structured bibliographical searches were made in the following databases: MEDLINE (PUBMED), Scopus, Web of Knowledge (WOK), IBECS, LILACS, SCIELO and grey literature.

Results: A total of nine scientific articles from different sources were included. The evidence levels were low. There are treatment and reincorporation programs which achieve promising results.

Med Segur Trab (Internet) 2014; 60 (235) 455-469

Key words: Treatment program, substance-related abuse, health personnel 


\section{INTRODUCCIÓN}

El uso de sustancias estimulantes es conocido desde la antigüedad ${ }^{1}$, y está presente en todas las culturas del globo. Podemos encontrar representaciones en tablillas de la antigua sumeria (3000 AC) de la planta de adormidera. Esta planta también se usaba en la antigua Grecia, en los templos de Esculapio, para tratar a los recién llegados con "un sueño sanador". Ejemplos como estos los tenemos a lo largo de la historia. En épocas más recientes (s. XVII) se empezó a preparar una tintura de opio llamada láudano, si esta se mezclaba con azúcar se denominaba vino de opio.

Ya en el siglo XIX llegó la gran revolución al conseguirse sintetizar fármacos puros. Muchos de ellos eran derivados de drogas conocidas desde hacía varios siglos, como el opio, la coca, o el café (entre otras plantas con efecto psicótropo). En laboratorios químicos, se sintetizaron la morfina (1805), codeína (1832), atropina (1833), cafeína (1841), cocaína (1860), heroína (1874), mescalina (1888) y los barbitúricos (1903). Todas estas sustancias tenían en principio una función médica, y desde la misma clase médica se ignoraban o minimizaban sus características adictivas en pos de ensalzar las curativas. Extendiéndose, popularizando y expendiéndose casi sin control hasta bien entrado el siglo XX, cuando se generalizan las restricciones en su dispensación y uso.

En 1961, la conferencia de las Naciones Unidas ${ }^{2}$ elaboró un convenio sobre el control de estupefacientes, que incluye aspectos como su elaboración, prescripción y dispensación. España lo ratificó publicándose en el BOE núm. 96/1966, de 22 de abril de 1962.

La última modificación de la legislación española sobre el control de algunas de estas sustancias está reflejada Real Decreto 1675/2012 ${ }^{3}$, de 14 de diciembre. En él se sigue manteniendo la necesidad de una receta adicional para la dispensación de estupefacientes (Convenio Único de 1961 sobre Estupefacientes de las Naciones Unidas), que engloba opioides y cannabinoides. No obstante no incluye al resto de sustancias susceptibles de abuso, tales como: benzodiacepinas, anfetaminas, agentes inhalantes y otras más o menos presentes en el medio sanitario.

Dentro de las sustancias de abuso en nuestro medio sanitario ${ }^{4}$ estarían los opioides tales como la morfina o el fentanilo. Ansiolíticos e hipnóticos tales como diazepam, lorazepam. Anfetaminas como el metilfenidato y agentes inhalantes como el óxido nitroso. Dentro de la población sanitaria están menos descritos los abusos con esteroides y anabolizantes. Desde 2012 se ha comenzado a comercializar un cannabinoide: dronabinol+cannabidol que en un futuro sabremos si es susceptible de adicción en nuestra población.

Según la encuesta 2007-2008 sobre consumo de sustancias psicoactivas 5 en el ámbito laboral en España (observatorio Español de drogas), dentro de la población general en edad laboral existen las siguientes prevalencias de consumo de sustancias: Alcohol: bebedores de riesgo $12.2 \%$ en hombres y $4.9 \%$ en mujeres y fármacos (referidos a ambos sexos) tales como tranquilizantes $6,7 \%$, somníferos $3,7 \%$, anfetaminas $0.9 \%$.

En torno al $12 \%$ de los médicos ${ }^{6}$ desarrollan a lo largo de su vida profesional (estimada en unos 40 años de ejercicio) alguna patología mental y/o adictiva. Según publicaciones en otros países la prevalencia de las adicciones en medio sanitario está en torno $8,5 \%$ en Francia ${ }^{7}$ y al $10-12 \%$ en USA $^{8}$.

Nuestra población de estudio son los sanitarios (médicos, odontólogos, farmacéuticos, enfermeras), ya que estos son los responsables de la prescripción, dispensación o administración de fármacos. Esta capacidad les da más fácil acceso a los fármacos que a la población general.

Este fácil acceso 9 facilita un posible abuso de fármacos, que no trae solo consigo el daño para la propia persona que lo realiza. También afecta a su entorno laboral, que tiene que asumir parte de la carga de trabajo del compañero ${ }^{9,12,13}$ Es un perjuicio económico para el sistema por el bajo rendimiento del trabajador, los errores del desempeño de sus 
tareas o los fármacos consumidos. No menos importantes son los daños a terceros, en forma de negligencias, con o sin denuncia. Las situaciones de abuso de fármacos en medio laboral normalmente se mantienen durante largos periodos de tiempo, haciéndose públicas normalmente por denuncias por mala praxis.

Se plantea la necesidad de una detección precoz de esta patología. Esto junto con una comunicación temprana al servicio de prevención de riesgos laborales correspondiente, podría hacer que se comenzara a tratar de forma precoz a estos profesionales. Esto podría apoyarse en el artículo $22^{10,11}$ de la ley de prevención de riesgos laborales, que incluye la posibilidad de realizar un reconocimiento médico obligatorio por un posible riesgo a terceros (los pacientes).

El trabajo en medio sanitario tiene como características negativas ${ }^{12}$ : jornadas prolongadas, turnos rotatorios, estrés, sobrecarga de trabajo. Esta realidad acarrea consecuencias físicas y mentales a los facultativos. Esto, junto con el hecho de la pérdida del miedo al uso fármacos (debido al conocimiento de sus mecanismos de acción) puede acabar en la decisión errónea de acudir a la automedicación para mantener el ritmo de trabajo.

Podemos sospechar ${ }^{12,13}$ que nuestro compañero consume sustancias por un cambio en sus conductas tales como disminución de la atención, accidentes laborales frecuentes y ausencias repetidas del puesto de trabajo sin causa evidente.

Este tipo de comportamientos es a menudo ocultado y tolerado por los propios compañeros. No debe ser fácil denunciar a un compañero ${ }^{14}$, aunque sus actos sean muy evidentes. Al detectar un caso, como se ha comentado anteriormente, se debería remitir al profesional a su servicio de prevención correspondiente, dado el peligro que puede suponer para la salud de los pacientes. Hay que intentar prevenir las negativas consecuencias derivadas del trabajo sanitario realizado bajo el abuso de fármacos.

En otros países existen programas ${ }^{14}$ para detectar al profesional enfermo, consistentes en entrenar a los compañeros para que detecten sospechas de abuso de fármacos y otras sustancias, e informar al servicio pertinente, que en nuestro país podría ser el servicio de prevención.

En los países anglosajones existen programas para rehabilitación ${ }^{12}$ y recuperación de estos profesionales. Hay variedad de intervenciones con o sin ingreso hospitalario, centros de día, comunidades terapéuticas. Muchos de ellos se basan en el programa de los 12 pasos que se inició en alcohólicos anónimos. En la mayoría de los casos los profesionales se incorporan a estos programas como alternativa a la inhabilitación.

En España existen programas de médico enfermo (Paime) ${ }^{6}$ y enfermera enferma (programa Retorn) ${ }^{15}$ gestionados por los colegios profesionales tanto de médicos y enfermeras correspondientes a la provincia donde esta colegiado el paciente. Ambas cuentan con una estructura similar en cuanto a su forma de actuación.

La comunidad de Madrid cuenta con un programa Paipse ${ }^{16}$, que trata a todos los profesionales sanitarios que trabajan en la red pública.

La forma de contacto del paciente con el programa es igual en todos los casos, y puede realizarse de tres maneras: comunicación directa del profesional enfermo, información confidencial de otro profesional al colegio profesional correspondiente o al Paipse y denuncia del colegio profesional correspondiente.

Todos los datos se manejan de la manera más confidencial ${ }^{6}$, evaluando el caso individualmente, proponiendo tratamiento psiquiátrico y/o ingreso hospitalario si fuera preciso. Todo esto llevado desde la más absoluta confidencialidad para el profesional enfermo.

Como se ha comentado anteriormente el tema de las adicciones en medio hospitalario es un tema ocultado por el sanitario adicto y sus compañeros, que por diversas causas no realizan denuncia a sus superiores o colegio profesional pertinente. Todo ello pese a las 
graves consecuencias que puede tener para la salud del paciente. A pesar de esto, existen pocos estudios que analicen en profundidad este tema. Este es el motivo por el cual nos inclinamos hacer esta revisión bibliográfica, centrándola en la situación actual y los resultados de los programas de rehabilitación en este colectivo de pacientes.

\section{MATERIAL Y MÉTODOS}

Se realizó una búsqueda de la literatura científica publicada en los últimos diez años (2003 a 2013) sobre el tratamiento del abuso de sustancias, especialmente fármacos, en el personal sanitario. Las bases de datos consultadas fueron: Pubmed (a través de MEDLINE), Scopus, Web of Knowledge (WOK), IBECS, LILACS y SCIELO. En otras búsquedas de literatura gris se consultaron: Dialnet, la página de internet del Plan Nacional contra drogas, Fundación Galatea y el $B O E$.

Se utilizaron los siguientes descriptores: "Substance-related abuse", "Healthpersonnel" y "Professional impairment". Se decidió utilizar este último descriptor para así poder obtener los trabajos que tratan al colectivo sanitario como pacientes y no como cuidadores de pacientes con adicciones, ya que se generaba mucho ruido en las búsquedas.

Con las búsquedas completadas, se realizó un análisis de la idoneidad de estos artículos para este trabajo, basándonos en el título y el resumen en aquellos que lo tenían. Se limitó a los diez últimos años para poder así ver cómo es la situación en la actualidad.

A los artículos seleccionados se les aplicó los correspondientes criterios de inclusión y de exclusión:

- Criterios de inclusión:

- Estudios realizados en humanos de ambos sexos.

- Estudios realizados en trabajadores del ámbito sanitario.

- Estudios sobre hábitos de consumo de sustancias.

- Estudios sobre el desarrollo de los programas de tratamiento y seguimiento.

- Estudios en los siguientes idiomas: Inglés, francés, castellano y portugués.

- Estudios publicados desde 2003 hasta 2013.

- Estudios transversales y de cohortes.

- Criterios de exclusión:

- Estudios centrados en drogas y/o sustancias no específicas del ámbito laboral (alcohol, tabaco, drogas ilegales).

- Cartas al director, artículos a propósito de un caso, revisiones bibliográficas.

- Estudios sobre abuso de sustancias en estudiantes universitarios de medicina y enfermería.

- Personal no sanitario que trabaja dentro del ámbito de la salud (celadores, administrativos, gerentes...).

- Artículos repetidos.

- Encuestas sobre incidencia/prevalencia de adicciones en el medio laboral.

\section{RESUMEN}

Con estas búsquedas se ha obtenido un total de 336 artículos, una vez eliminados los artículos duplicados se realizó un análisis de pertinencia y posteriormente se aplicaron los criterios de exclusión y de inclusión. Finalmente se obtuvo una colección de nueve artículos. 


\begin{tabular}{|c|c|c|c|c|c|}
\hline $\begin{array}{c}\text { Base } \\
\text { de datos }\end{array}$ & Descriptores & Términos libres & $\begin{array}{l}\text { Estrategia de } \\
\text { búsqueda }\end{array}$ & Resultados & $\begin{array}{c}\text { Resultados finales tras } \\
\text { confirmar utilidad y } \\
\text { criterios de exclusión e } \\
\text { inclusión }\end{array}$ \\
\hline $\begin{array}{l}\text { MEDLINE- } \\
\text { Pubmed }\end{array}$ & $\begin{array}{l}\text { "Professional } \\
\text { Impairment", } \\
\text { "Substance- } \\
\text { Related } \\
\text { Disorders, } \\
\text { Health } \\
\text { Personnel" }\end{array}$ & $\overline{\text { Peer }}$ & $\begin{array}{l}\text { "Professional } \\
\text { Impairment" } \\
\text { [Mesh] AND } \\
\text { "Substance- } \\
\text { Related } \\
\text { Disorders } \\
\text { "[Mesh] AND } \\
\text { "Health } \\
\text { Personnel" } \\
\text { [Mesh] } \\
\text { - }\end{array}$ & $\begin{array}{l}174 \\
- \\
19\end{array}$ & $\frac{6}{0}$ \\
\hline LILACS & & $\begin{array}{l}\text { "Inhalant abuse" } \\
\text { "Substance- } \\
\text { Related } \\
\text { Disorders", } \\
\text { "physician } \\
\text { impairment" }\end{array}$ & $\begin{array}{l}\text { "Inhalant abuse" } \\
\text { Or "Substance- } \\
\text { Related } \\
\text { Disorders" } \\
\text { AND "Physician } \\
\text { Impairment" }\end{array}$ & 3 & 1 \\
\hline SCOPUS & $\begin{array}{l}\text { "Impairedphy- } \\
\text { sician", "health- } \\
\text { personnel" }\end{array}$ & $\begin{array}{l}\text { Nurses, "subs- } \\
\text { tance abuse" } \\
- \\
\text { "substance-rela- } \\
\text { ted disorders", } \\
\text { "physicians } \\
\text { impairment" }\end{array}$ & $\begin{array}{l}\text { "impaired } \\
\text { physician" } \\
\text { or "nurse" } \\
\text { or " health } \\
\text { personnel" and } \\
\text { "substance } \\
\text { abuse" } \\
\text { - } \\
\text { "substance- } \\
\text { related } \\
\text { disorders" AND } \\
\text { "physicians } \\
\text { impairment" }\end{array}$ & $\frac{50}{85}$ & $\frac{1}{-}$ \\
\hline $\begin{array}{l}\text { Búsqueda } \\
\text { manual }\end{array}$ & & & & & 1 \\
\hline
\end{tabular}

A los artículos seleccionados se les aplicó el checklist para selección de artículos, y se observó que cumplen los criterios STROBES.

\section{RESULTADOS}

A pesar de la heterogeneidad de los datos encontrados en los artículos, se realizó la siguiente tabla: 


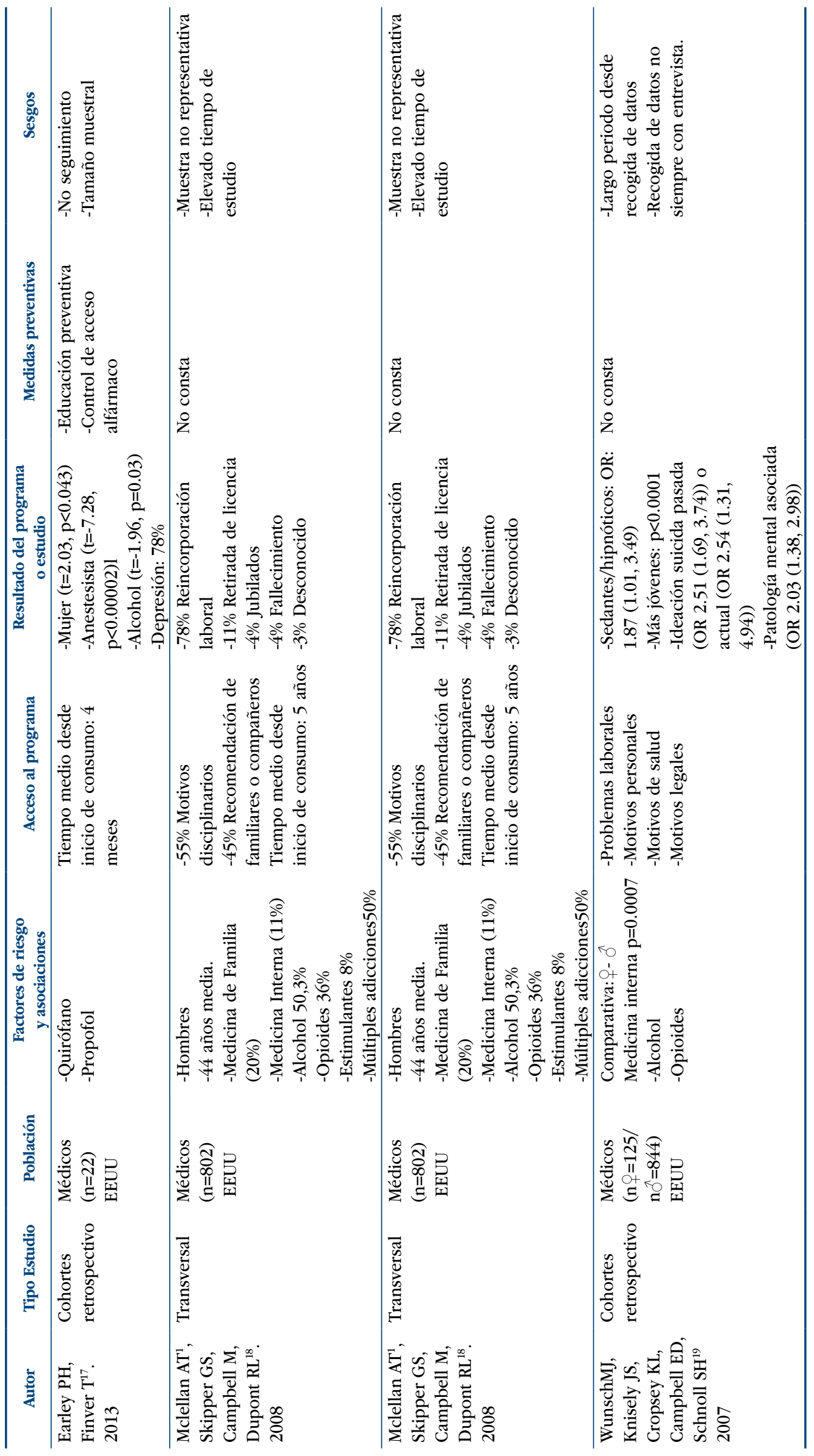



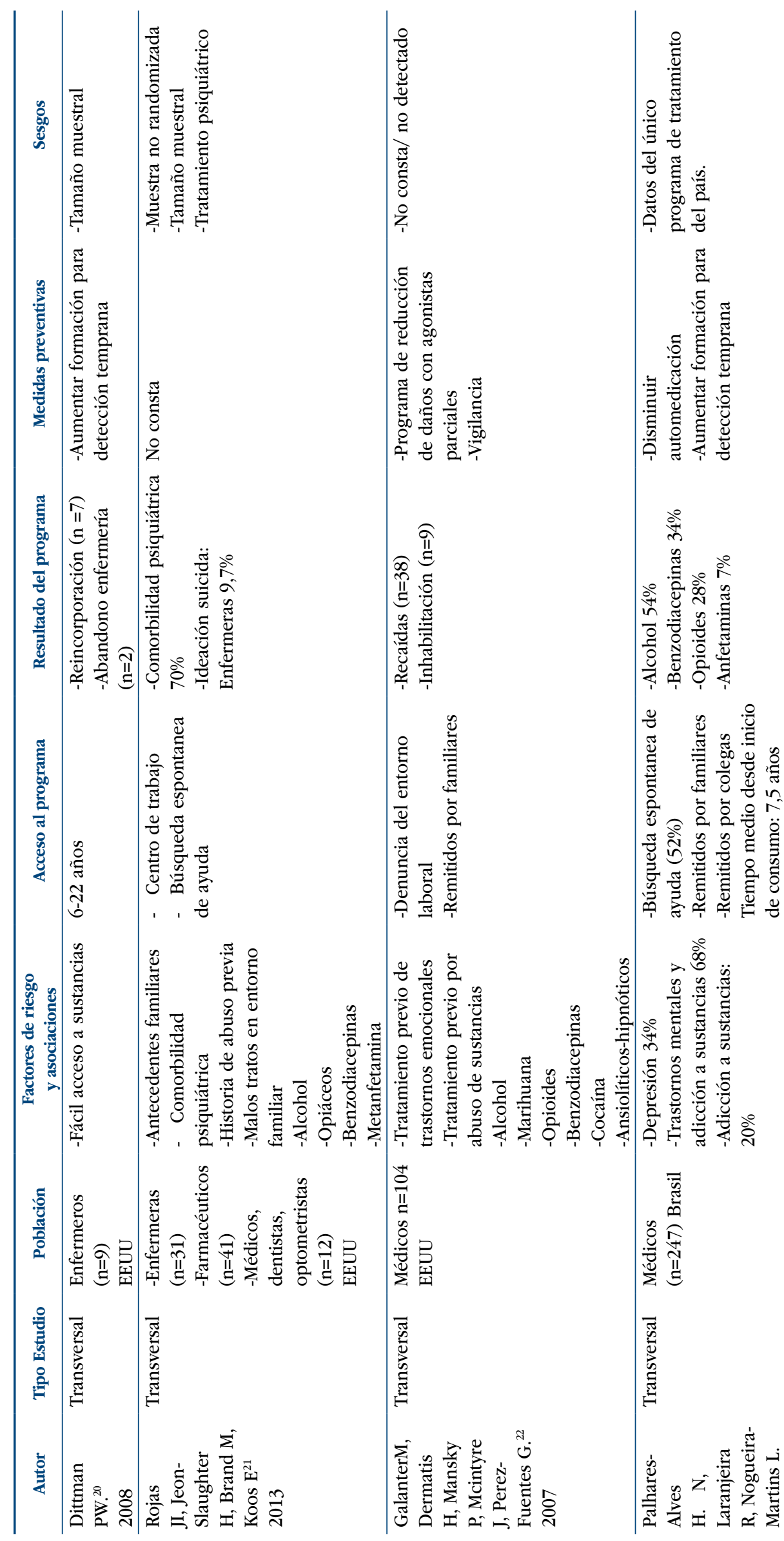

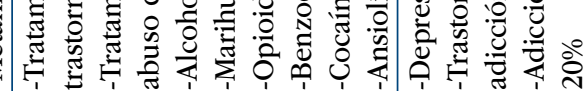

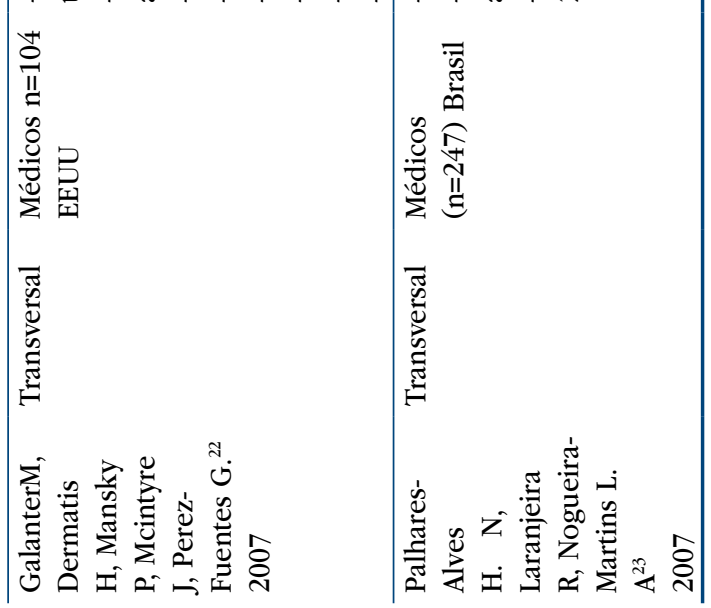




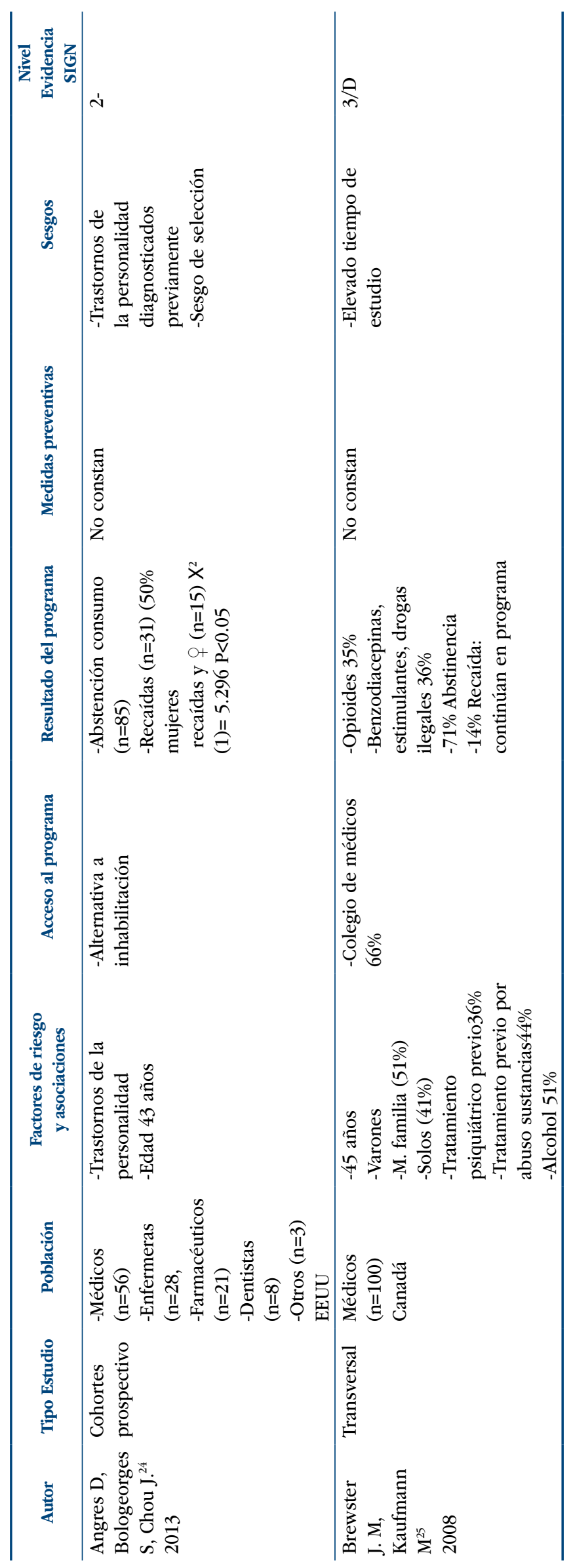


En el estudio de Earley $\mathbf{P} \mathbf{H}^{17}$ se obtuvieron los datos de consumo de propofol de una muestra más amplia de médicos de un programa de tratamiento de adicciones. Se compararon ambos grupos con el test de la $t$ de student. Se observó que en todos los casos había un acceso laboral al propofol (todo personal de quirófano). Esta era la droga de elección en el 50\% de los casos. Otro 20\% acabó abusando principalmente de este fármaco después de haberse iniciado con alcohol o fentanilo.

Se pudo observar un mayor consumo en mujeres. El inicio del abuso se debía a la necesidad de conciliar el sueño o debido a motivos de ansiedad. Se pudo observar una alta prevalencia de patología mental previa al consumo, así como abusos en la infancia.

Es de resaltar el poco tiempo que transcurre desde el inicio del consumo hasta que acuden al programa de tratamiento, una media de 4 meses. Esto se asocia a las dramáticas consecuencias del consumo de propofol.

Mclellan AT ${ }^{18}$ valora la eficacia de 16 de los 49 programas estatales de salud americano en el tratamiento del médico con adicción. En este estudio se vio que había un $87 \%$ de población masculina. Las especialidades más frecuentes fueron medicina de familia, medicina interna y anestesiología Las drogas o fármacos de abuso más habituales eran alcohol 50\%, opioides $36 \%$ y estimulantes $8 \%$. El policonsumo estaba presente en el $50 \%$ de los casos. Más de la mitad de los pacientes del programa acudían de forma obligatoria, el resto por sugerencia de familiares, colegas o del centro de trabajo.

El 70\% fueron ingresados directamente en comunidades terapéuticas. El 95\% de los programas incluían o se basaban en el método de los 12 pasos. Un 32\% de los pacientes necesitaron tratamiento frente a un trastorno ansioso depresivo. De los 802 médicos $19.3 \%$ no pudieron completar el tratamiento o la supervisión, de estos un 55\% dejó de ejercer voluntariamente, 48 fueron inhabilitados, 22 fallecieron (6 suicidios) durante el periodo de tratamiento-seguimiento. De los 8 restantes no se tienen datos. Eligieron prolongar voluntariamente el periodo de seguimiento 67 trabajadores. Fue obligado prolongarlo en 132 debido a las recaídas (126) o por falta de cumplimiento del contrato terapéutico.

A los cinco años, el $78.7 \%$ estaba trabajando sin ningún tipo de restricción. Un 4\% había dejado de trabajar voluntariamente, $11 \%$ fueron inhabilitados y otro $4 \%$ había fallecido.

Se concluyó que este tipo de programas es efectivo en la rehabilitación de los médicos.

En un programa de tratamiento y seguimiento de médicos con adicciones, Wunsch MJ ${ }^{19}$ comparó los perfiles de aspectos psicosociales y de abuso de sustancias por géneros de 996 médicos (125 mujeres). Se recogieron datos de 1995 a 1998, con una entrevista en el momento de la admisión en el programa. Se incluyeron datos demográficos, uso de fármacos y/o drogas, historia psicosocial, historia psiquiatría e historia médica. Se compararon los grupos usando los test de Chi-cuadrado y $t$ de student.

No se encontraron diferencias por género en la droga de abuso primaria, a excepción de los sedantes/hipnóticos, más habituales en mujeres. Las preferidas en ambos grupos fueron alcohol y opioides.

En el grupo de mujeres se encontró el doble de patología mental asociada, en ambos grupos depresión mayor, trastornos distímicos y trastorno bipolar fueron los más frecuentes. Las ideas o intentos de suicidio se presentaban más en el grupo de mujeres. También había más frecuencia de historia psiquiátrica familiar.

En el estudio de Dittman ${ }^{20}$ nos presentan una muy pequeña muestra de enfermeros que superan un programa de rehabilitación $(n=9)$. No específica sustancia, (narcóticos). En este estudio el autor destaca que estos enfermeros eran considerados por sus compañeros como personas muy perfeccionistas e muy inteligentes. El Uso de los fármacos se realizaba principalmente como forma de evasión. Las conductas se mantuvieron adictivas se mantuvieron entre 6 a 22 años. Como resultado final todos 
tuvieron una rehabilitación satisfactoria, aunque dos no llegaron a reincorporarse por miedo a una nueva recaída.

En el artículo de Rojas $^{21}$ nos presenta un estudio cohortes retrospectivo sobre un grupo de sus pacientes $(n=84)$ que han superado un programa de rehabilitación, pasando una nueva evaluación mediante un cuestionario clínico estándar y un segundo cuestionario sobre trastornos psiquiátricos (PAI), con el fin de buscar asociación entre abuso de sustancias y trastornos psiquiátricos. Se divide la población en tres grupos: enfermería(n=31), farmacéuticos $(n=41)$ y un tercero de profesionales que pueden prescribir $(n=12)$. Con los datos estadísticos se aplica test de Wilkonxony los valores PAI con T-score, hallando una $\mathrm{p}<0.05$.

Entre sus hallazgos del cuestionario clínico: la media de edad era uniforme entre los tres grupos ( $\mathrm{x}=45$ años). El grupo de farmacéuticos es el de un mayor recorrido profesional (media 20 años), menor en enfermeras (10 años). Presenta diferencias de géneros entre los tres grupos, con una fuerte feminización entre el grupo de enfermería (77\% vs $25 \%$ resto de grupos), y las sustancias de abuso principal varían según el grupo: benzodiacepinas en enfermería (32,26\%), farmacéuticos opioides (83\%). Llama la atención la presencia de antecedentes familiares de abuso a sustancias en el $61 \%$ de enfermería (vs $41 \%$ media del estudio) Los tres grupos habían recibido tratamientos previos por adicciones $(\mathrm{X}=1.53)$ : con el programa 12 pasos $(73 \%)$ u otros tratamientos con ingreso (22.5\%). Preferido este último por el grupo de enfermería.

La comorbilidad psiquiátrica está más presente en el grupo de enfermería (87\%), tales como depresión mayor $(48,4 \%)$ e ideación suicida en el anterior mes $9.7 \%$ (no presente este último en los otros dos grupos).

El estudio de Galanter $^{22}$ trabaja con una muestra de 104 médicos que han superado un programa de rehabilitación. Los datos preceden de cuestionario clínico rellenado al inicio del programa, resultados de monitorización de orinas aleatorias, progreso de las sesiones del programa de 12 pasos, trabajo supervisado por monitor. Estas dos últimas se clasificaban como satisfactorio o insatisfactorio para análisis estadístico posterior. Como sustancias de consumo: alcohol (63\%, como consumo único 36\%) opiáceos (34\%), y otras sustancias como marihuana, benzodiacepinas, cocaína, hipnóticos, alucinógenos o estimulantes.

La muestra está compuesta principalmente por médicos varones (76\%) empleados en el momento de la intervención (63\%),

Los autores analizan las recaídas dentro del programa $(n=38)$ hallando más recaídas con consumos previos de cocaína (68\% vs 23\%), desempleados (55\%) Nueve pacientes fueron inhabilitados y expulsados del programa. Como resultado de la variables estudiadas los autores destacan 3 variables como predictores reales del éxito de los programas: experiencia del programa basado en los 12 pasos, tener empleo a la llegada del programa y los meses que se permanece en el programa después de la admisión.

Los autores sugieren para mejorar el éxito de estos programas añadir tratamiento de reducción de daños con agonistas parciales (buprenorfina)

En el trabajo de Palhares-AlvesH. $\mathbf{N}^{23}$ se estudian las características de los trabajadores que acuden a un programa de apoyo psiquiátrico a los médicos del estado de Sao Paulo (Brasil). Este programa trata a los médicos que acuden con patología mental y/o con abuso de sustancias. Se realizaron entrevistas que recogían los datos socio-demográficos, ocupacionales, de abuso de sustancias y psiquiátricos con criterios ICD-10.

Se comprobó que el 52\% acudían de forma espontánea al programa, un 32\% por recomendaciones de familiares y el resto por recomendaciones de los compañeros. La edad media fue de 42.4 años, pero el problema de adicción había comenzado 7.5 años antes.

De los trabajadores que acudieron un $20 \%$ fue exclusivamente por abuso de fármacos-drogas y un $67 \%$ por asociar este consumo a una patología mental. La sustancia 
de la que más se abusó fue el alcohol (54\%) seguido de los fármacos como benzodiacepinas (34\%) opioides (28\%) y anfetaminas (7\%). Un 31,2\% había estado previamente en tratamiento psiquiátrico.

Se consideró relevante la observación de que el $78 \%$ de los médicos del programa se automedicaban. Como medidas de prevención secundaria se propone la formación en escuelas médicas para la detección temprana de abuso de fármacos entre compañeros y disminuir el hábito de automedicación.

El artículo de Angres $\mathbf{D}^{24}$, es un estudio cohortes de pacientes que han concluido un programa de rehabilitación intensivo (6-8 semanas) en régimen de centro de día, basado en el programa de los 12 pasos y un seguimiento posterior durante 2 años. Durante el tratamiento se valora a los pacientes con dos cuestionarios psiquiátricos "MillonClinicalMultiaxialInventory" (MCMI-1) y "Temperament and CharacterinventoryRevised" (TCI-R)y cuestionario clínico estándar. Al final de los dos años se evaluó a los pacientes separándolos en dos grupos: abstinentes $(n=85,73.3 \%)$ y recaídas $(n=31$, 26.7\%). se analizaron las variables cualitativas mediante $X^{2} \cdot$ La prevalencia de los trastornos de personalidad encontrados (35.3\%), según al MCMI-I estaban presentes de forma similar en ambos grupos, no encontrando asociación significativa entre ambos $X^{2}(1)=0.210$, $\mathrm{P}=0.666$. Las variables más significativas analizadas fueron el sexo, dependencia alcohólica previa. Encontrando una asociación significativa entre las recaídas y el género femenino $(\mathrm{n}=15) \mathrm{X}^{2}(1)=5.296 \mathrm{P}<0.05$.

La asociación entre la variable recaída y la dependencia alcohólica $\left(X^{2}(8)=9.603\right.$, $\mathrm{P}<0.294)$ fue una asociación negativa para mantenimiento de la abstinencia, y fue una variable predictiva independiente de recaída entre el grupo del trastorno de personalidad antisocial: $\left(X^{2}(3)=9.042 P<0.029\right)$.

Brewster J. $\mathbf{M}^{25}$ estudia a los 100 primeros médicos que fueron admitidos a un programa de tratamiento y monitorización de sustancias (centro de desintoxicación + periodo de monitorización: grupos de apoyo, visitas periódicos, muestras aleatorias de orina). En los casos de recaídas se continuaba con el programa si esta era leve, si eran severas o se empezaba el tratamiento completo o se abandonaba el programa. Los datos se analizaron estadísticamente.

El 90\% eran hombres, con una media de edad de 40 años. La mitad eran médicos de familia o médicos generales. Un $41 \%$ ejercían solos, y el alcohol era la drogas elección en el $51 \%$ de los casos. El 37\% tenía consumo de opioides y el 36\% benzodiacepinas, estimulantes o drogas ilegales.

El $71 \%$ no tuvo recaídas, pero un $14 \%$ tuvo que reiniciar el tratamiento por este problema. El 85\% acabó satisfactoriamente el programa y recuperó su licencia sin ningún tipo de limitación.

\section{DISCUSIÓN}

Los artículos seleccionados fueron publicados entre los años 2007 y 2013. Sin embargo las fechas de acceso a los programas de tratamiento son poco homogéneas, abarcando un periodo desde 1995 hasta 2011. Este hecho, como sugieren McLellan $\mathrm{AT}^{18} \mathrm{y}$ WunschMJ ${ }^{19}$, puede suponer que haya habido un cambio en los hábitos de consumo de fármacos en los estudios con un largo periodo de tiempo.

Las profesiones desempeñadas por los pacientes con adicciones de estos estudios encontramos: médicos, enfermeras, farmacéuticos y en menor medida odontólogos. Dentro de los médicos las especialidades más frecuentemente estaban presentes los artículos analizados fueron Medicina de familia/general, medicina interna, cirugía y anestesia. ${ }^{18,19,21,22,23,25}$ En el artículo de Earley $\mathrm{PH}^{17}{ }^{17}$ toda la población de estudio procedía del área quirúrgica. 
Todos los estudios presentan medias de edades similares, entre 40-45 años. Respecto al género, en todos los artículos había una elevada representación masculina, variando la media entre el $68 \%$ y el $90 \%$, salvo dos excepciones. El artículo de Earley ${ }^{17}$ muestra un porcentaje del $45 \%$ de mujeres, frente al $26 \%$ de la base de datos de médicos general. La otra excepción es el estudio de Rojas $\mathrm{JI}^{21}$ que separa la población en tres grupos, según profesión. En el grupo de enfermería -profesión tradicionalmente femenina- hay una mayor presencia de mujeres en este grupo de tratamiento.

En España los resultados publicados por el programa Galatea de los años 2010-2012, en referencia a personal médico, concuerdan con respecto a las especialidades médicas con mayor representación entre los médicos atendidos en este programa (MFyC, anestesia, sin especialidades) y las edades predominantes, mayor representación masculina (58.8\%), predominancia de consumo de alcohol (20\%) y tóxicos (26.5\%) aunque estos últimos no se desglosan por tipo ${ }^{6}$.

Solo dos de los artículos han estudiado los antecedentes personales psiquiátricos, estando estos presentes entre $36 \%-47 \%^{18,22}$. En el Programa Galatea atienden a un $11.8 \%$ de los pacientes con patología dual, y otros diagnósticos de salud mental 38,2\%

Más investigados han sido los tratamientos previos por adicciones a fármacos y/o sustancias, variando entre 37\%-64\% ${ }^{21,22,23,25}$. Uno de los ítems analizados en el artículo de Rojas $^{21}$ fue el número de tratamientos previos por adicciones. Siendo la media de 1.53, con una desviación estándar de 1.60.

En tres artículos se encontraron referencias alos antecedentes personales de abusos tanto físicos, sexuales y/o psicológicos. Dos de estos estudios hacen referencia a los abusos sufridos durante la infancia: en el estudio de Early P. H et al ${ }^{17}$ hay un elevado porcentaje en el grupo de mujeres (61\%); en el estudio de Wunsch $\mathrm{MJ},{ }^{20}$ se encontraron diferencias significativas tanto en el abuso psicológico (OR1.60; 1.07-2.38) como sexual durante la infancia (OR 6.22; 3.25-10.31).

Dos de los artículos seleccionados hacen referencia a los antecedentes familiares (AF), psiquiátricos y de consumo de sustancias (alcohol/drogas). En el artículo de Wunsch $\mathrm{MJ}^{19}$ había más posibilidad de encontrar este tipo de AF, siendo la diferencia estadísticamente significativa en los antecedentes familiares de patología mental (OR=1.85 (1.22; 2.79)). En el estudio de Rojas $\mathrm{JI}^{21}$ el grupo de enfermería, por otra parte muy feminizado, fue el que tuvo una frecuencia mayor tanto de antecedentes familiares de patología mental como AF de abuso de alcohol/drogas. (26\% y $61.2 \%$ respectivamente).

Los programas de tratamiento que se han seguido en los estudios analizados son descritos solo en cinco de nuestros artículos ${ }^{18,21,22,24,25}$. Estos consisten en una primera parte un programa intensivo de duración variable con o sin ingreso, en comunidades terapéuticas. Posteriormente seguimiento durante 5 años reuniones periódicas dentro o fuera del programa (grupos de apoyo), monitorización en trabajo, analíticas de orina aleatorias. Varios ${ }^{22,24}$ de ellos incluyen el programas de 12 pasos para el programa de mantenimiento, el estudio de Angres D et $a l^{24}$ desde el inicio del programa.

En todos los artículos estudiados la sustancia de abuso predominante fue el alcohol, en un alto porcentaje asociado a policonsumo con fármacos. La excepción la encontramos en el artículo de Early ${ }^{17}$, en el que hay una correlación negativa entre el consumo de propofol y alcohol. Al comparar estos datos con los del programa Retorn ${ }^{15}$ en España, observamos que en este último el consumo de alcohol o fármacos es inferior (12\%-4\%) y el programa Galatea $23,5 \%$ tóxicos y $20 \%$ consumo perjudicial de alcohol.

Las siguientes sustancias que encontraron abuso fueron opioides, benzodiacepinas, anfetaminas/estimulantes y otras drogas ilegales. En este orden excepto en el estudio de Palhares-Alves H. N. et $a l^{23}$, en el que había un mayor porcentaje de consumo de benzodiacepinas.

La forma de acceso a los programas de tratamiento es mayoritariamente a instancias del ámbito laboral, generalmente para evitar una inhabilitación forzosa. Una excepción la 
encontramos en el estudio de Palhares-Alves HN et al ${ }^{23}$, donde la mayoría de médicos acceden en busca de ayuda de forma espontánea. No hay uniformidad en las siguientes formas de acceso, que suelen ser bien por recomendación familiar o de compañeros de trabajo. Cuatro estudios ${ }^{17,18,20,23}$ dan la información sobre el tiempo que transcurre entre el inicio del consumo y el momento en el que acuden al programa de tratamiento. Debido a las características del propofol, este grupo lleva consumiendo una media de 4 meses en el momento de acceder al programa de tratamiento. Esto es algo que contrasta con los 5 y 7,5 años de los trabajos de McLellanAT ${ }^{18}$ y Palhares-Alves $\mathrm{HN}^{23}$ respectivamente. Dittman ${ }^{20}$ describe un intervalo temporal desde 6 a 22 años.

En dos de nuestros artículos los autores intentan analizar si existe asociación entre trastornos de personalidad y adicciones. En el artículo de Angres $\mathrm{D}^{24}$.los autores encuentran una prevalencia de un $35.3 \%$ de trastornos de personalidad. Un $24.4 \%$ tiene criterios para más de un trastorno de personalidad, sin diferencias estadísticas entre los dos grupos del estudio.

Esto contrasta con los resultados de Rojas ${ }^{21}$ cuyos hallazgos son solo de $1.19 \%$ de alteraciones psiquiátricas considerando los tres grupos de pacientes. Si consideramos solamente al grupo de enfermería, esta patología estaría presente en un $3.23 \%$ de los pacientes.

Esta discordancia de resultados estaría presente también si comparamos la ansiedad entre Rojas $^{21}$ y Angres $^{24}$ (3.5\%-49.8\%). Sin embargo el programa Retorn ${ }^{26}$ en España obtiene unas cifras de ansiedad del $71 \%$. No obstante estos estudios si tienen concordancia de resultados cuando valoran depresión mayor o dependencia alcohólica (alrededor del 39\% y $60 \%$ respectivamente en los tres trabajos).

Los datos aportados sobre reincorporación laboral después superado el programa ${ }^{18,23,25}$ presentan valores que oscilan entre $74-85 \%$, En los estudios en los que no se había terminado a fecha de recogida de datos a terminar el programa de seguimiento se observó contaban con una adherencia $97 \%^{21}-73 \%^{24}$

En aquellos estudios en los que ya se había finalizado el periodo de seguimiento la reincorporación laboral se produjo en el 85\% de los casos (McLellan AT $^{18}$ )

Los resultados obtenidos sobre recaídas en la adicciones durante el periodo de seguimiento son muy heterogéneos, variando desde el $15.7 \%^{18}$ hasta el $-37 \%^{22}$. En la mayor parte de los casos estos pacientes siguieron en tratamiento.

\section{CONCLUSIONES}

Una de las dificultades encontradas en la realización de este artículo ha sido los escasos artículos publicados en los últimos diez años sobre este tema. Estos estudios presentan sesgos. Uno de los más importantes es el pequeño tamaño muestral en muchos de los artículos seleccionados.

Los trabajos de nuestro estudio están publicados desde 2003 hasta 2010. En varios de estos artículos sus bases de datos se remontan a varios años atrás, algo que puede afectar a los hábitos de consumo. Tampoco hay que olvidar que continúan apareciendo en el mercado nuevos fármacos susceptibles de abuso. Estos factores nos indican que los hábitos de consumo han podido haber cambiado o evolucionado en el momento actual.

Como se ha comentado en la introducción existen programas de tratamiento y rehabilitación del profesional enfermo ${ }^{6,15,16}$. En nuestro país los programas están gestionados por los colegios profesionales correspondientes. Además del PAIPSE en la Comunidad de Madrid, que está integrado en el servicio madrileño de salud. Estos modelos tienen sus pros y contras. Por una parte al estar en los colegios profesionales pueden acceder a estos programas los profesionales enfermos trabajen en el sistema 
público o privado, y en contra, los servicios de prevención cuando detecten un caso pueden tener menor comunicación con los programas.

Los datos obtenidos por estos programas no están siendo debidamente difundidos, suponemos que por la especial sensibilidad de los datos obtenidos, lo que nos impide conocer la situación real de estos programas tanto en nuestro país como a nivel internacional.

Como manera de detección temprana de estos profesionales enfermos, se debería concienciar a los compañeros sanitarios de la importancia de comunicación precoz de conductas o sospechas a los servicios de prevención pertinentes.

\section{BIBLIOGRAFIA}

1. Torres A, Santo Domingo J, Pascual F, Freixa F, Álvarez C. Historia de las adicciones en la España contemporánea, [monografía en internet] plan nacional sobre drogas; 2009, disponible en: http://www. pnsd.msc.es/Categoria2/publica/pdf/HistoriaAdicciones_EspContem.pdfmiguel.

2. Convenio único de 1961 sobre estupefacientes. Boletín Oficial del Estado n. ${ }^{\circ}$ 96/1966 (22-04-1966) [internet fecha de acceso 15-01-2014] disponible en: http://www.judicatura.com/Legislacion/1667.pdf.

3. Real Decreto $1675 / 2012$, de 14 de diciembre, por el que se regulan las recetas oficiales y los requisitos especiales de prescripción y dispensación de estupefacientes para uso humano y veterinario. Boletín Oficial del Estado número 313(29-12-2013) [internet, fecha de acceso 20-01-2014] Disponible en: http:// www.caib.es/sacmicrofront/archivopub.do?ctrl=MCRST353ZI138432\&id=138432.

4. Diaz L, Ulloa C, Taubert de Freitas F, Amorin L. [El uso de drogas en el personal de enfermería]. Cienc. Enferm 2011;17(2): 37-45

5. Observatorio Español sobre Drogas. Encuesta 2007-2008 sobre consumo de sustancias psicoactivas en el ámbito laboral en España. [monografía en internet fecha de acceso 15-01-2014] disponible en: http:// www.pnsd.msc.es/Categoria2/observa/pdf/Encuesta2007-2008AmbitoLaboral.pdf.

6. Fundación Galatea. El Programa de Atención Integral al Médico Enfermo y el control del ejercicio profesional [monografía en internet]. Barcelona. Fundación Galatea. (fecha de acceso 15-01-2014) disponible en http://www.fgalatea.org/pdf/paimm.pdf.

7. Institutnational de prévention et d'éducationpour la santé. [sede web] Des substancespsychoactives plus consomméesdanscertainssecteurs de travail,16-01-2012 [fecha de acceso 15-01-2014] disponible en: http:// www.inpes.sante.fr/30000/actus2012/004.asp.

8. Flaherty JA, Richman JA. Substance use and addiction among medical students, residents, and physicians PsychiatrClin North Am. 1993 Mar;16(1):189-97.

9. Chavignaud I, Coscas S, Lafaye G. [The consumption of subtances in hospital, defence or temptation?]. Rev Infirm. 2012 Nov;(185):16-18.

10. Ley 31/1995 de 8 de Noviembre, de Prevención de Riesgos Laborales. Boletín Oficial del Estado, número 269, de 10-11-95.amj.a2098.

11. Agulló Hernández D. [Treatment program in Spain for nurses suffering from depression and drug addiction]. RevInfirm. 2012 Nov;(185):27. 